\title{
Eradication of Pseudomonas aeruginosa Biofilms Using the Combination of $n$-butanolic Cyclamen coum Extract and Ciprofloxacin
}

\author{
Morvarid Shafiei ${ }^{1}$; Ahya Abdi Ali ${ }^{1,}$; Fereshteh Shahcheraghi ${ }^{2}$; Azra Saboora ${ }^{1}$; Kambiz \\ Akbari Noghabi ${ }^{3}$ \\ ${ }^{1}$ Department of Biology, Faculty of Science, Alzahra University, Tehran, IR Iran \\ ${ }^{2}$ Department of Bacteriology, Pasteur Institute of Iran, Tehran, IR Iran \\ ${ }^{3}$ Department of Molecular Genetics, National Institute of Genetic Engineering and Biotechnology, Tehran, IR Iran \\ *Corresponding author: Ahya Abdi Ali, Department of Biology, Faculty of Science, Alzahra University, Tehran, IR Iran. Tel/Fax:+98-2188058912, E-mail: abdialya@alzahra.ac.ir \\ Received: September 14, 2013; Revised: November 30, 2013; Accepted: December 28, 2013
}

\begin{abstract}
Background: Biofilm formation is a major pathogenic factor in different bacteria such as Pseudomonas aeruginosa. A number of studies have reported that bacterial biofilms show different levels of antibiotic resistance. In order to re-sensitize the bacterial biofilms to antibiotics, biofilms should be dispersed.

Objectives: In this study, the effect of $n$-butanolic Cyclamen coum extract in combination with ciprofloxacin was examined on one, three and five day old P. aeruginosa biofilms. The synergistic effect of $n$-butanolic C. coum extract and ciprofloxacin towards dispersing preestablished P. aeruginosa biofilms was also studied.

Materials and Methods: The ability of biofilm formation by six different P. aeruginosa strains was confirmed by microtiter plate method and PCR assay for the cupA gene. The extraction of C. coum tubers was achieved by fractionation method using different solvents. The minimum inhibitory concentration (MIC) of $n$-butanolic C. coum extract and ciprofloxacin against planktonic cells was evaluated using agar well diffusion and microdilution methods. The microdilution chequerboard method was used to determine the fractional biofilm eradication concentration index (FBCI), when the combination of $n$-butanolic $C$. coum extract and ciprofloxacin were used against $P$. aeruginosa biofilms.

Results: The ability of biofilm formation by P. aeruginosa strains was quantitatively confirmed. The PCR method confirmed the existence of cup A gene (172 bp) in all studied strains. Saponin content of the $n$-butanolic C.coum extract was $156 \mu \mathrm{g} / \mathrm{mL}$. The extract revealed antibacterial activity against planktonic cells of $P$. aeruginosa strains. The results showed that one and three day old biofilms are affected by either ciprofloxacin or $n$-butanolic C. coum extract. However, $n$-butanolic C. coum extract in combination with ciprofloxacin was significantly more effective against $P$. aeruginosa biofilms.

Conclusions: Using $n$-butanolic C. coum extract in combination with ciprofloxacin offers a novel strategy to control biofilm-based infections caused by P. aeruginosa.
\end{abstract}

Keywords:Pseudomonas aeruginosa; Biofilm; Cyclamen coum

\section{Background}

Biofilms are organized communities of sessile bacteria that grow on different surfaces. Bacteria can develop biofilms on submerged surfaces such as natural aquatic systems, living tissues, tooth surfaces, indwelling medical devices and implants. One of the most medically important biofilm-forming species is Pseudomonas aeruginosa, which is commonly associated with lung infections in patients with cystic fibrosis. Biofilm formation by $P$. aeruginosa occurs through several stages including, attachment to the surface by surface flagella and proteins (such as cup A), followed by division to form microcolonies, and lastly maturation including expression of matrix polymers. Sessile bacteria display phenotypes and possess properties that are considerably different from planktonic cells (1). They are much more resistant to antimicrobial treatments and as a result, biofilms can cause particularly devastating chronic infections or facilitate life-threatening nosocomial infections in short time courses.

Considering many biofilm-related problems, it is clear that finding an effective and safe medication with biofilm dispersal properties is one of the important issue in clinical microbiology and infectious disease (2). Once a biofilm has been dispersed, the planktonic bac- 
teria become rapidly susceptible to the conventional antibiotics. Therefore, various treatments such as different vaccines, antibiotics and interferon gamma have been studied for biofilm dispersal properties. However, active constituents such as plant extracts may be a better source for biofilm dispersion (3).

Plant-based medicines and derivatives not only have effective roles in the treatment of biofilm-based diseases but also, have fewer side effects in comparison to synthetic medicines. The use of plant extracts and phytochemicals, with antimicrobial and biofilm dispersal properties, can be of great significance in therapeutic treatments. In the last few years, a number of studies have been conducted in different countries to prove such capacities. Many plants have been used because of the compounds they synthesize in their secondary metabolism.

Cyclamen coum is a perennial understory herb from the Myrsinaceae family, which is endemic to the forests of North of Iran. According to the Ahmadbeigi et al study, the tubers of $C$. coum contain large amounts of saponins and they can be extracted and accumulated in organic phase using different solvents such as petroleum ether, 70\% ethanol and $n$-buthanol (4).

Saponins are a diverse group of compounds widely distributed in the plant kingdom, which are characterized by their structure containing a triterpene or steroid aglycone and one or more sugar chains. Saponins as commercially significant compounds, have expanding applications in food, cosmetics, and pharmaceutical industries due to their anticancer, antioxidant, antihypertensive, antibacterial, antifungal and antiviral activities (5).

\section{Objectives}

The aim of the present study was to elucidate the dispersal property of $n$-butanolic C. coum extract in combination with ciprofloxacin toward pre-formed P. aeruginosa biofilms.

\section{Materials and Methods}

\subsection{Bacterial Strains}

Four clinical strains which had been used in previous studies, P.A 214, P.A 25, P.A 29 and P.A 50, along with two standard strains, $P$. aeruginosa PAO1 and P. aeruginosa ATCC $8821 \mathrm{M}$, were used in this study (6). Frozen stocks were prepared in trypticase soy broth containing $20 \%$ glycerol. Strains were sub-cultured weekly on nutrient agar and stored at $4^{\circ} \mathrm{C}$.

\subsection{Biofilm Formation Assay}

A modified microtiter plate test was used to determine the biofilm formation. An overnight culture of each strain was grown in trypticase soy agar plus $0.2 \%$ glucose (Merck, Germany) for 20 hours at $37^{\circ} \mathrm{C}$. The bacterial suspensions were prepared in trypticase soy broth plus $0.2 \%$ glucose medium according to 0.5 McFarland turbidity standard as measured by absorbance (0.08 - 0.1 at $625 \mathrm{~nm}$ ) using a spectrophotometer (Schimadzu, model UV-120-01, Japan). Bacterial suspensions $(200 \mu \mathrm{L})$ were aliquoted in to a 96-well polystyrene microtiter plate (Sigma Aldrich, St. Louis, Missouri, USA) and incubated at $37^{\circ} \mathrm{C}$ for 24 hours without agitation.

After 24 hours of incubation at $37^{\circ} \mathrm{C}$, the content of each well was aspirated, and each well was washed with sterile physiological saline to remove all nonadherent cells. The attached bacteria were fixed with absolute methanol for 10 minutes. The plates were stained using crystal violet $(1 \% \mathrm{~W} / \mathrm{V})$. Excess stain was washed off and the plates were rinsed with tap water. The bound dye was resolubilized with $200 \mu \mathrm{L}$ of glacial acetic acid $(33 \%, v / v)$. The OD of each well was measured at $570 \mathrm{~nm}$ using an ELISA reader. Uninoculated wells containing media served as blanks. A 3-grade scale was used to evaluate the ability of biofilm formation: Negative: ODs < 0.500; Positive: ODs 0.500-1.500; Strongly positive: ODs $>1.500$. All tests were carried out in triplicates (7).

\subsection{Detection of cup A Gene}

DNA was prepared by boiling lysis of the bacteria. The cupA gene sequence, which was provided from the Pseudomonas Genome Database (http://www.pseudomonas.com), utilized for designing primer sequences using the primer3 version 7 software.

The primers (Forward -5 - CTACCGCTATTCCACCGAAG-3` and reverse-5 -AGGAGCCGGAAAGATAGAGG-3`) were used in this study.

PCR: amplification of targeted DNA was carried out in $25 \mu \mathrm{L}$ reaction volumes, each containing $2 \mathrm{mM} \mathrm{MgCl}_{2}$, $250 \mu \mathrm{M} \mathrm{dNTP}, 0.4 \mu \mathrm{M}$ (each) primer, $1 \mathrm{U}$ of Taq polymerase (Invitrogen, Carlsbad, Calif.), and $10 \mu \mathrm{L}$ of whole-cell bacterial lysate, and adjusted to $25 \mu \mathrm{L}$ by adding highperformance liquid chromatography-grade $\mathrm{H}_{2} \mathrm{O}$.The amplification was carried out in Thermo Cycler (Merck Company, Germany). After an initial denaturation for 5 minutes at $94^{\circ} \mathrm{C}, 40$ cycles were completed, each consisted of 40 seconds at $95^{\circ} \mathrm{C}, 45$ seconds at $59.4^{\circ} \mathrm{C}$ (annealing temperature), and 60 seconds at $72^{\circ} \mathrm{C}$. A final extension of 7 minutes at $68^{\circ} \mathrm{C}$ was applied. The PCR products were analyzed on $1 \%$ agarose gels, visualized under UV illumination and photographed.

\subsection{Saponin Extraction}

Tubers of C. coum were collected from the Golestan Forest (Golestan Province in Iran). Freshly collected tubers of $C$. coum were dried in the shade, sliced into small pieces and pulverized using a mechanical grind- 
er. The dried powder (100 g) was successively extracted using petroleum ether (300 $\mathrm{mL})$ and $70 \%$ ethanol (300 $\mathrm{mL}$ ) using the Soxhlet apparatus (Bio Thechnics, India). After 24 hours, the extract was fractionated by $n$-buthanol in to two phases. Lower phase ( $n$-butanolic) which contained cyclamen saponins was dried by a Rotavapor (Buchi Flawil, Switzerland). The solid precipitate was dissolved in dimethyl-sulphoxide (DMSO), taking in to account that the maximum concentration of DMSO in the test solution should not exceed one percent (4).

\subsection{Determination of Total Saponin Content}

Total saponin content was determined by the vanillin-sulfuric acid method. n-butanolic C. coum extract was mixed with vanillin ( $8 \%, \mathrm{w} / \mathrm{v})$ and sulfuric acid (72\%, $\mathrm{w} / \mathrm{v})$. The mixture was incubated at $60^{\circ} \mathrm{C}$ for 10 minutes followed by cooling in an ice water bath for 15 minutes. The absorbance was measured at $538 \mathrm{~nm}$. All determinations were carried out in triplicates. Saponin $(1 \mathrm{mg} /$ $\mathrm{mL}$ ) (Merck, Germany) was used to determine the calibration curve (8).

\subsection{Determination of Antibacterial Activity of the Extract}

The agar well diffusion method was used for evaluation of antibacterial activity of $n$-butanolic C. coum extract. About $100 \mu \mathrm{L}$ of microbial suspension $\left(1 \times 10^{8}\right.$ $\mathrm{CFU} / \mathrm{mL}$ ) was mixed with molten Muller Hinton agar medium and poured into sterile Petri dishes. Nine wells were made in each $15 \mathrm{~cm}$ plate. Thereafter, different concentrations of $n$-butanolic C. coum extract were added in three wells of each plate. Negative and positive controls were $100 \mu \mathrm{L}$ of diluted PBS and ciprofloxacin, respectively. After overnight incubation at $37^{\circ} \mathrm{C}$ the inhibition zones were measured.

\subsection{Antibacterial Activity of Ciprofloxacin against $P$. aeruginosa Strains}

Minimum inhibitory concentrations (MICs) and minimum bactericidal concentrations (MBCs) of ciprofloxacin (Sigma-Aldrich) were determined for six P. aeruginosa strains using the broth microdilution method according to the procedures outlined by the Clinical Laboratory Standards Institute (CLSI) guidelines.

\subsection{Biofilm Dispersion Assay}

In this study, the effects of $n$-butanolic C. coum extract with or without ciprofloxacin on one, three and five day old biofilms were determined. As mentioned in section 3.2, biofilms were established in microtiter plates (Sigma Aldrich, St. Louis, Missouri, USA). After 24, 72 or 120 hours, the media were discarded from the wells and the plates were washed thoroughly with PBS ( $\mathrm{pH}$
$=7.4$ ), to remove unattached cells. Thereafter, checkerboard arrangements of $n$-butanolic $C$. coum extract and ciprofloxacin were made and added to the pre-formed biofilms. In the checkerboard technique, two drugs are placed in microtiter wells with drug concentrations equal to, above and below their MIC. In our study, the chosen ciprofloxacin concentrations were from 0.25 to $64 \mu \mathrm{g} / \mathrm{mL}$ and selected $n$-butanolic $C$. coum extract concentrations were from 50 to $500 \mu \mathrm{g} / \mathrm{mL}$.

Medium (trypticase soy broth plus $0.2 \%$ glucose) alone was added to a subset of the wells to serve as the control. Each microtiter plate contained three wells as sterility controls, three wells as growth controls, nine wells for different concentrations of $n$-butanolic $C$. coum extract and antibiotic alone and the combination of the $n$-butanolic $C$. coum extract and antibiotic. The plates were incubated for 24 hours at $37^{\circ} \mathrm{C}$ and stained with crystal violet $(\mathrm{CV})$. The fractional biofilm eradication concentration index (FBECs) was determined for the combination of $n$-butanolic C. coum extract and antibiotic as follows:

$$
\begin{aligned}
& \text { FBECA }=\operatorname{MBECA}(\mathrm{c}) / \operatorname{MBECA}(\mathrm{a}), \\
& \mathrm{FBECB}=\operatorname{MBECB}(\mathrm{c}) / \operatorname{MBECB}(\mathrm{a}) \\
& \Sigma \text { FBEC }=\text { FBECA + FBECB }
\end{aligned}
$$

Where MBEC denotes minimum biofilm eradication concentration, subscripts A and B denote antibiotic and $n$-butanolic C. coum extract, subscripts in parentheses, (c) and (a) denote the activity measurements in combination and alone, respectively. The sum of both FBECs was used to classify the combination of $n$-butanolic extract and ciprofloxacin as follows: Synergistic ( $\Sigma$ FBEC $\leq 0.5)$, partially synergistic $(0.5<\Sigma$ FBEC $\leq 1)$, indifferent $(1<\Sigma$ FBEC $\leq 4)$, or antagonistic ( $\Sigma$ FBEC $>$ 4) (10).

\subsection{Statistical Analysis}

Data analysis was performed using Excel 2010 software. The results of the experiment were expressed as mean \pm standard deviations (SD) for three replicates in each test. The significance of all the data was tested with the Student's t-test $(\mathrm{P}<0.05)$ using Excel 2010 software.

\section{Results}

Microtitre plate and PCR methods were used to determine the biofilm forming capabilities of $P$. aeruginosa strains. The results revealed that $P$. aeruginosa strains PA25 and PA29 were weakly positive (0.5 - 0.7 OD), P. aeruginosa PAO1 and PA 50 were moderately positive (1.3-1.5 OD) and P. aeruginosa strains 8821M and PA214 (1.7-1.8 OD) were strongly positive in biofilm formation. It seems that, these differences are due to the diversity of matrix materials. According to Ghafoor et al. study, highly mucoid P. aeruginosa (such as $8821 \mathrm{M}$ and PA214) produces biofilms with alginate origin matrix where- 
as, non-mucoid $P$. aeruginosa (such as PAO1) produces biofilms with Psl, eDNA and proteins origin matrix (3). As shown in Figure 1, all strains examined in this study, contained cupA gene with a resulting fragment of 172 bp.

The type of organic solvents had a profound effect on artifact formation as a result of extraction and improved the rates of isolation of the saponins from cyclamen tubers. According to standard curve, the saponin content of the $n$-butanolic $C$. coum extract was $156 \mu \mathrm{g} / \mathrm{mL}$. The $n$-butanolic $C$. coum extract revealed antibacterial activity against planktonic $P$. aeruginosa. The data pertaining to the antimicrobial potential of the $n$-butanolic $C$. coum extract and ciprofloxacin are presented in Table 1.

Figure 2 shows the effect of ciprofloxacin (CIP) or $n$-butanolic $C$. coum extract as well as their combination (CIP + plant extract) on one day old biofilm of six strains of $P$. aeruginosa. The antibiotic (at $4 \times \mathrm{MIC}$ ) and $n$-butanolic C. coum extract (at $74 \mu \mathrm{g} / \mathrm{mL}$ ) affected one day old biofilm mass but their combination had substantial effect on $P$. aeruginosa biofilm mass $(\mathrm{P}<0.05)$.

The ciprofloxacin did not affect three day old biofilm mass at all concentrations. The $n$-butanolic $C$. coum extract at $140 \mu \mathrm{g} / \mathrm{mL}$ eliminated three day old $P$. aeruginosa biofilms. However, $n$-butanolic $C$. coum extract (at $95 \mu \mathrm{g} / \mathrm{mL}$ ) in combination with ciprofloxacin (at $4 \times$ MIC) dramatically dispersed three day old preestablished biofilm (Figure 3). The ciprofloxacin, $n$-butanolic C. coum extract and their combination did not significantly affect five day old biofilm mass (data not shown). By checkerboard synergy technique, $n$-butanolic C. coum extract in combination with ciprofloxacin exhibited synergistic effect against one and three day old $P$. aeruginosa biofilms ( $\Sigma$ FBEC $\leq 0.4$ )(9).

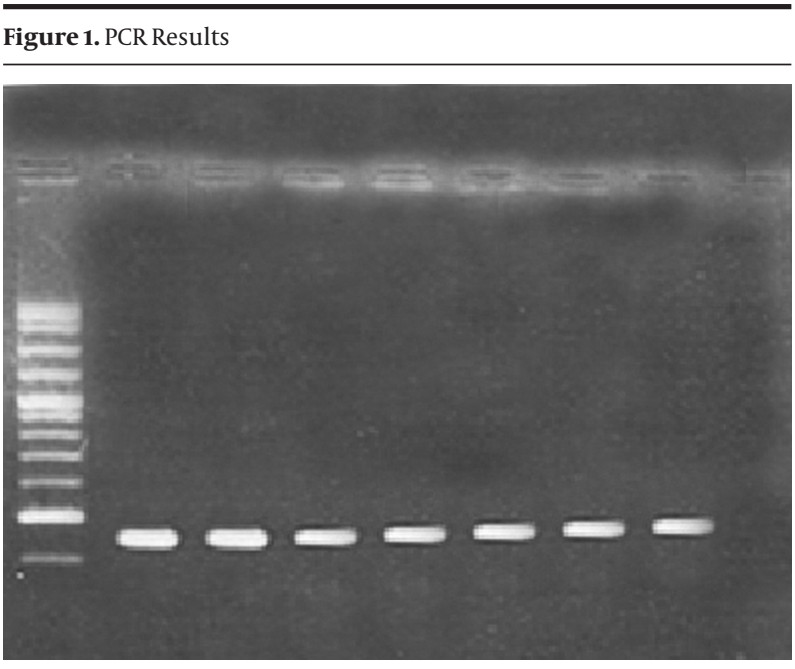

From left to right: Marker (M), Positive control (1), PAO1 (2), 8821M (3), PA214 (4), PA25 (5), PA29 (6), PA50 (7) and Negative control (8). The PCR product is $172 \mathrm{bp}$.
Figure 2. The Effect of Ciprofloxacin (CIP), n-butanolic C. coum Extract (Extract) and the Combination of Ciprofloxacin and $n$-butanolic $C$. coum Extract (CIP+extract) on one day old Biofilm of Six P. aeruginosa Strains

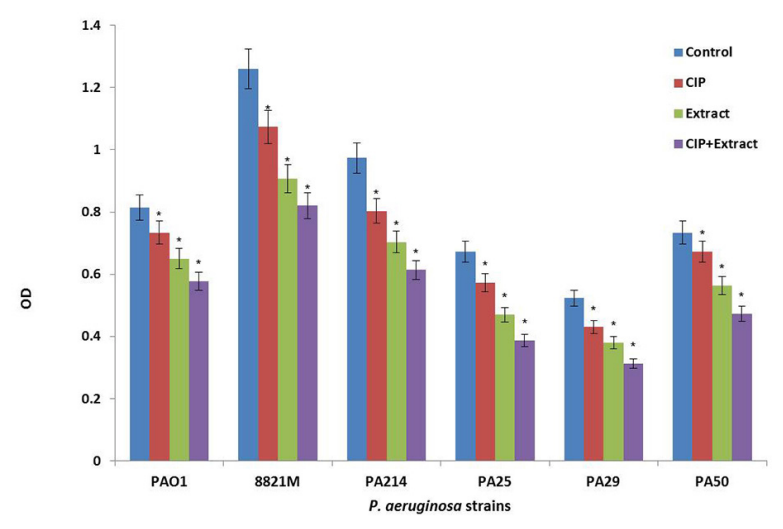

Bars represent the mean OD570 values, and error bars represent the standard deviations. Blue bars, control samples treated with TSB medium alone; red bars, samples treated with ciprofloxacin (CIP); green bars represent biofilms treated with $n$-butanolic $C$. coum extract (Extract); purple bars represent biofilms treated with the combination of ciprofloxacin and $n$-butanolic C. coum extract (CIP+Extract). Statistical analysis was done using the Student's t-test the P value $<0.05$ was considered as sig nificant (noted with *).

Figure 3. The Effect of Ciprofloxacin (CIP), n-butanolic C. coum Extract (Extract) and the Combination of Ciprofloxacin and $n$-butanolic C. coum Extract (CIP+Extract) on three day old Biofilm of six P. aeruginosa Strains

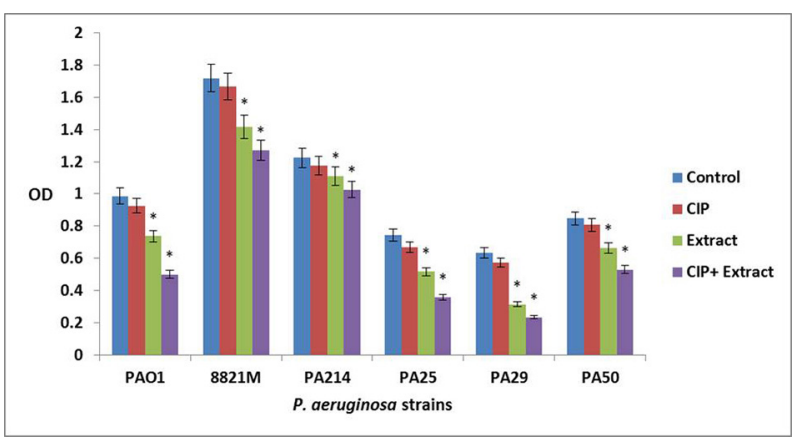

Bars represent the mean OD570 values and error bars represent standard deviations. Blue bars, control samples treated with TSB medium alone; red bars, samples treated with ciprofloxacin (CIP); green bars represent biofilms treated with $n$-butanolic $C$. coum extract (Extract); purple bars represent biofilms treated with the combination of ciprofloxacin and nbutanolic C. coum extract (CIP+Extract). Statistical analysis was done using Student's t-test and the P value $<0.05$ was considered as significant (noted with $*$ ). 
Table 1. Minimum Inhibitory Concentration (MIC) of $n$-butanolic C. coum Extract and Ciprofloxacin Using Agar Well Diffusion and Microtiter Plate Methods According to the CLSI Protocols.

\begin{tabular}{llll}
\hline $\begin{array}{l}\text { P. aeruginosa } \\
\text { Strains }\end{array}$ & $\begin{array}{l}\text { n-butanolic } C \\
\text { coum extract }\end{array}$ & \multicolumn{2}{c}{ Ciprofloxacin } \\
\cline { 2 - 4 } & MIC, $\boldsymbol{\mu g} / \mathbf{m L}$ & MIC, $\boldsymbol{\mu g} / \mathbf{m L}$ & MBC $^{\text {a }}, \boldsymbol{\mu g} / \mathbf{m L}$ \\
\hline PAO1 & 256 & 0.25 & 0.5 \\
$\mathbf{8 8 2 1 M}$ & 256 & 1 & 2 \\
$\mathbf{2 1 4}$ & 254 & 4 & 8 \\
$\mathbf{2 5}$ & 254 & 8 & 16 \\
$\mathbf{2 9}$ & 257 & 8 & 16 \\
$\mathbf{5 0}$ & 257 & 4 & 8 \\
\hline a Minimum Bactericidal Concentration &
\end{tabular}

\section{Discussion}

The emergence of infections due to highly resistant $P$. aeruginosa, which is capable of forming biofilms, causes a significant problem in the medical field, today. The healthcare community is not able to effectively treat such infections with the old classes of antibiotics, which rapidly lose their efficacy. Effective treatments for the elimination of established biofilms could diminish the morbidity and subsequently reduce healthcare costs associated with nosocomial infection (1).

In recent years, the molecules that control bacterial biofilms through nonmicrobicidal mechanisms have gained a lot of utility and attention. These molecules can act synergistically with conventional antibiotics to overcome infectious diseases (1). The antibiofilm agents will disperse bacterial biofilms, while the antibiotics will eliminate the bacterial population. Therefore, it is essential to determine how conventional antibiotics affect the ability of antibiofilm agents to control biofilm perpetuation (2). For this reason, we studied the ability of $n$-butanolic $C$. coum extract to disperse bacterial biofilms in the presence of an approved antibiotic such as ciprofloxacin. At first, biofilm-forming ability of six different $P$. aeruginosa strains was investigated using microtiter plate and PCR (for the detection of adhesion genes) methods. All tested strains were able to form biofilms (Figure 1). According to Vallet et al. the CupA system plays a fundamental role in biofilm formation. They confirmed that the CupA-dependent adhesions are more required at an earlier stage of biofilm formation than type IV pili (10).

To quantify any synergistic effects between $n$-butanolic C. coum extract and ciprofloxacin toward eliminating biofilm colonization, pre-formed P. aeruginosa biofilms were treated with the combination of $n$-butanolic $C$. coum extract and ciprofloxacin. In this study, ciprofloxacin was investigated because it is widely prescribed to treat $P$. aeruginosa infections. In the current study, the combination of ciprofloxacin and plant extract showed a drastic effect towards dispersion of one and three day old $P$. aeruginosa biofilms $(\mathrm{P}<0.05)$. A 2 -fold drop and a 4 -fold drop in MIC were noted with ciprofloxacin, when one and three day old biofilms were pretreated with $n$-butanolic C. coum extract. Therefore, high levels of synergy were observed when low concentrations of $n$-butanolic $C$. coum were used in combination with the antibiotic. However, compared with the samples treated with medium only, this combination did not affect the five day old biofilm mass (in all concentrations examined). It seems that this phenomenon is due to the overproduction of alginate and over thickening of the biofilm mass.

The exact mechanism of synergy is currently unknown and further work is required to reveal the role and effect of cellular and molecular parameters on biofilm dispersion to extend the benefits of this synergy. Based on the observation that $n$-butanolic C. coum extract contains high levels of saponins, it is reasonable to assume the extract may re sensitize resistant bacteria to the antibiotics by inhibiting cell-to-cell communication (Quorum sensing). It has been revealed that quorum sensing has significant role in biofilm formation and the production of virulence factors. Quorum-sensing systems are important targets to address the sensitivity of bacteria to antibiotic compounds and the host immune system (11). By using $n$-butanolic $C$. coum extract, quorum sensing was inhibited and eventually the biofilm was dispersed and bacterial population was eliminated by ciprofloxacin. While these results are based on the analysis of crude, unfractionated extracts, they are also the first of many steps towards the development of new drugs with biofilm dispersal property.

In conclusion, it was demonstrated that the combination of an antibiofilm agent containing saponin compounds with conventional antibiotics provides an effective strategy for remediating biofilm based diseases.

\section{Acknowledgements}

We thank the Vice Chancellor of Alzahra University for their financial support.

\section{Authors' Contribution}

None declared.

\section{Financial Disclosure}

Upon completion of the study, all authors had no conflict of interest.

\section{Funding/Support}

This study was part of the Ph.D thesis supported by Alzahra University. 


\section{References}

1. Hall-Stoodley L, Stoodley P. Biofilm formation and dispersal and the transmission of human pathogens. Trends Microbiol. 2005;13(1):7-10.

2. Petrova OE, Sauer K. Dispersion by Pseudomonas aeruginosa requires an unusual posttranslational modification of BdlA. Proc Natl Acad Sci US A. 2012;109(41):16690-5.

3. Ghafoor A, Hay ID, Rehm BH. Role of exopolysaccharides in Pseudomonas aeruginosa biofilm formation and architecture. Appl Environ Microbiol. 2011;77(15):5238-46.

4. Ahmadbeigi Z, Saboora A. Comparison of three methods for saponin extraction from tuber of Cyclamen coum Miller. $J$ Sci. 2009;21(2):27-38.

5. Oyekunle MA, Aiyelaagbe OO, Fafunso MA. Evaluation of the antimicrobial activity of saponins extract of Sorghum bicolor L. Moench. AfrJ Biotechnol. 2006;5(23).

6. Abdi-Ali A, Mohammadi-Mehr M, Agha Alaei Y. Bactericidal activity of various antibiotics against biofilm-producing Pseudomonas ae- ruginosa. Int JAntimicrob Agents. 2006;27(3):196-200.

7. Pitts B, Hamilton MA, Zelver N, Stewart PS. A microtiter-plate screening method for biofilm disinfection and removal. J Microbiologic Methods. 2003;54(2):269-76.

8. Hassan SM, Al Aqil AA, Attimarad M. Determination of crude saponin and total flavonoids content in guar meal. Advance Med Plant Res. 2013;1(2):24-8.

9. Khani-Juy Abad F, Abdi-Ali A, Gharavi S. Antibiofilm activities of certain biocides in Pseudomonas aeruginosa. Iran J Microb. 2009;1(4):23-7.

10. Vallet I, Olson JW, Lory S, Lazdunski A, Filloux A. The chaperone/ush er pathways of Pseudomonas aeruginosa: identification of fimbrial gene clusters (cup) and their involvement in biofilm formation. Proc Natl Acad Sci US A. 2001;98(12):6911-6.

11. Rogers SA, Huigens RW 3rd, Cavanagh J, Melander C. Synergistic effects between conventional antibiotics and 2-aminoimidazole-derived antibiofilm agents. Antimicrob Agents Chemother. 2010;54(5):2112-8 